\title{
Irmãos de bebês recém-nascidos internados em unidades de neonatologia: revisão bibliográfica
}

Infant sisters and brothers of newborns hospitalized in neonatology units: bibliographic review

Hermanas y hermanos infantes de recién nacidos hospitalizados en unidades de neonatología: revisión bibliográfica

Valeria Alejandra Silva-Quintul ${ }^{1}$ (D) https://orcid.org/0000-0002-3644-6456

Carola Rosas ${ }^{1,2}$ (D) https://orcid.org/0000-0001-7693-3278

Patricia Triviño-Vargas ${ }^{1}$ (1) https://orcid.org/0000-0002-6910-5092

Tatiana Emperatriz Victoriano-Rivera ${ }^{1}$ (D) https://orcid.org/0000-0002-2403-5756

\section{Resumo}

Objetivo: Analisar a importância das visitas de irmãos de recém-nascidos internados em Unidades de Neonatologia a partir das experiências relatadas na literatura.

Métodos: Para proporcionar uma reflexão sobre as experiências no mundo, é realizada uma revisão de artigos que contenham a temática dos irmãos, selecionados pela amplitude do estudo, seja quantitativo, qualitativo ou experiência mista.

Conclusão: A importância da implementação da visita de irmãos nos diversos centros hospitalares reside na sua detecção como fator fundamental para a redução do estresse parental e familiar, melhorando o vínculo entre os irmãos e a comunicação dentro da família. Os entraves residem no risco de transmissão de microrganismos a pacientes com sistema imunológico imaturo ou debilitado, juntamente com as políticas internas de muitos centros, que impedem a entrada de crianças pequenas como visitantes.

\section{Abstract}

Objective: To analyze the importance of visits by sisters and brothers of newborns hospitalized in Neonatology Units from the experiences reported in the literature.

Methods: To provide a reflection on the experiences in the world, a review of articles containing the theme of siblings is carried out, selected for the breadth of the study, whether it is quantitative, qualitative or mixed experience. Conclusion: The importance of the implementation of sibling visits in the various hospital centers lies in its detection as a fundamental factor that reduces parental and family stress, improving the bonds between siblings and communication within the family. The impediments lie in the risk of transmission of microorganisms to patients who have an immature or weakened immune system, together with the internal policies of many centers, which prevent the entry of young children as visitors.

\section{Resumen}

Objetivo: Analizar la importancia de las visitas de hermanas y hermanos de los recién nacidos hospitalizados en las Unidades de Neonatología desde las experiencias que se relatan en la literatura.

Métodos: Para proporcionar una reflexión de las experiencias en el mundo, se realiza una revisión de artículos que contienen la temática de hermanos, seleccionados por amplitud del estudio ya sea experiencia cuantitativa, cualitativa o mixta.

Conclusión: La importancia de la implementación de las visitas de los hermanos en los diversos centros hospitalarios radica en su detección como un factor fundamental que permite disminuir el estrés parental y familiar, mejorando los lazos entre hermanos y la comunicación en el interior de la familia. Los impedimentos radican en el riesgo de transmisión de microorganismos a pacientes que poseen un sistema inmune inmaduro 0 debilitado, junto con las políticas internas de muchos centros, que impiden el ingreso de niños pequeños como visitas.

\section{Descritores}

Irmãos, Neonatologia; Infecções; Enfermagem pediátrica

\section{Keywords}

Siblings; Neonatology; Infections;

Pediatric nursing

\section{Descriptores}

Hermanos; Neonatología; Infecciones; Enfermería pediátrica

\section{Como citar:}

Silva-Quintul VA, Rosas C, Triviño-Vargas P, Victoriano-Rivera TE. Irmãos de bebês recém-nascidos internados em unidades de neonatologia: revisão bibliográfica. Rev Soc Bras Enferm Ped. 2021;21(1):51-6. 


\section{Introdução}

O cuidado dos recém-nascidos hospitalizados evoluiu. No início, as unidades permitiam pouco tempo para os pais permanecerem na unidade, sendo ainda mais restrito para os outros famíliares, pois estava relacionado ao risco de transmissão de infecções para os recém-nascidos, priorizando este sobre o contato afetivo. Atualmente, tem sido progressivamente demonstrado que a inclusão da família ${ }^{(1)}$ não é um risco adicional de infecções per se, e que favorece a continuidade do vínculo familiar e o subsequente cuidado do bebê.

Primeiro foi incluída a visita da mãe, principalmente para a amamentação, depois os pais e avós, mas ainda há uma desfasagem no caso dos irmãos. A forma como a família é incluída na recuperação dos recém-nascidos possui grande variabilidade, ${ }^{(2)}$ de acordo com as características de infraestrutura, número e preparação do pessoal, além das condições do recém-nascido prematuro.

São evidentes efeitos na sua melhoria, com a presença da mãe e do pai, mas a inclusão dos irmãos poderia ter repercussões na dinâmica familiar após a alta, tais como menores níveis de estresse dos pais e melhores laços de apego entre os irmãos. ${ }^{(3)}$

No Chile, as experiências são variadas, e cada centro de saúde, segundo seu regulamento, permite ou restringe a visita de irmãos. O Ministério de Saúde, recomenda a programação de visitas de irmãos e outros membros da família em caso de hospitalizações prolongadas. ${ }^{(4)}$ No entanto, é uma decisão de cada centro realizá-lo ou não, e há pouca documentação das experiências no país. Por outro lado, as características dos usuários neonatais sempre levam ao medo do risco de infecções, portanto, as visitas tendem a ser limitadas.

Esta pesquisa é parte dum projeto para incluir a família nas visitas aos neonatos hospitalizados na unidade de neonatologia de um hospital no Chile e corresponde a uma revisão bibliográfica da literatura disponível a fim de analisar as evidências e refletir sobre as mudanças na concepção do significado da hospitalização para uma criança (irmã/irmão) e sua família.

\section{Métodos}

Com o objetivo de proporcionar uma reflexão das experiências no mundo, foi realizada uma revisão dos artigos contendo a temática dos irmãos de bebês recém-nascidos internados em unidades de neonatologia. Os artigos foram selecionados por data de publicação, abrangência do estudo, desenho quantitativo, qualitativo ou misto.

Foram selecionados os descritores irmãos, neonatologia, infecções. Foram incluídas bases de dados de texto completo, como Science Direct, e bases de dados de referência como Medline (PubMed), Scopus para a busca. Todos os artigos utilizados estão disponíveis gratuitamente em texto completo.

A apresentação dos resultados é feita considerando cinco eixos, que foram identificados de forma dedutiva, após a leitura de todos os trabalhos. O eixo da pandemia foi incluído porque é o cenário mais restritivo nas visitas hospitalares.

Foram revisados 45 artigos, 36 dos quais foram incluídos neste estudo, por apresentarem maior afinidade com o objetivo desta revisão.

\section{Discussão}

\section{Evolução do significado da hospitalização do recém-nascido para uma criança e sua família}

Desde seu aparecimento na França em 1880 com a invenção da incubadora, a neonatologia é uma área em contínua evolução. Os conhecimentos, equipamentos, tratamentos e técnicas introduzidos nos cuidados contribuíram para a diminuição da taxa de mortalidade neonatal. Atualmente, o foco também está em garantir uma qualidade de vida adequada no futuro. ${ }^{(1)}$

Entretanto, a hospitalização de uma criança é uma situação complexa, e em muitos casos inesperada, que confronta a família com um processo cheio de dúvidas dificultando a previsão do seu curso. Isso afeta especialmente os pais, e é de se esperar que eles sofram alguma alteração de humor, como "sintomas de ansiedade e estresse através de sentimentos de medo e pavor devido aos eventos vivenciados na Unidade de Terapia Intensiva Neonatal (UTIN)"'.(5)

Os pais têm a autoridade ética e legal para a tomada de decisão sobre o estado de saúde de seus filhos, eles poderiam incorporar-se na tomada de decisão em conjunto com profissionais, ${ }^{(6)}$ portanto, eles devem 
ser informados de possíveis condições adversas ou diagnósticos para os recém-nascidos e suas famílias de forma prática, por sua vez, a equipe de saúde deve permitir que os pais participem do "estabelecimento de metas, decisões, transições e planos para o futuro", considerando quais resultados são importantes para eles, ${ }^{(7)}$ pois muitas vezes esperam resultados diferentes da equipe de saúde, relacionados com a qualidade da assistência prestada. ${ }^{(8)}$

A principal preocupação de ambos os pais é o estado de saúde da criança, além do estresse experimentado na UTIN e a angústia pela separação com os outros filhos. A participação da mãe durante a internação é considerada fundamental para a recuperação e evolução do recém-nascido, o que implica longas estadias hospitalares, obrigando-a a se distanciar do resto das crianças e da organização familiar. ${ }^{(9)}$ Deve-se considerar que a assistência dos pais na UTIN é influenciado não só pela presença de outras crianças no lar, mas também por limitações pessoais como tempo de trabalho, distância do hospital e despesas de viagem. ${ }^{(10)}$

Desta forma, os pais, especialmente a mãe, são o eixo central em torno do qual são desenvolvidas as diversas estratégias destinadas a promover a obtenção de melhores cuidados, desenvolvimento e uma melhor qualidade de vida no futuro para os recém-nascidos e suas famílias. Relacionado a isso, achamos a expressão do Cuidado Centrado na Família (FCC), que corresponde numa abordagem da assistência médica baseada na associação de usuários, famílias e profissionais da saúde, cujo objetivo é capacitar a família do paciente promovendo a participação direta no cuidado dele. (11) "A participação como cuidador é diversa e muda de acordo com a condição médica do bebê". ${ }^{(12)}$

Atualmente existem modelos de cuidados que formalizam a implementação de cuidados centrados na família, que apresentam semelhanças e diferenças entre eles, sendo fundamentais para sua concepção, avaliação, implementação e expansão, a liderança conjunta dos pais. ${ }^{(13)}$

A inclusão do restante do grupo familiar tem sido mais restrita, como é o caso dos irmãos, que suportam de acordo com sua idade as diversas consequências produto da hospitalização precoce devido a alguma condição grave ou prematuridade do irmão recém-nascido, portanto é necessário considerar esta opção no cuidado da saúde, uma vez que sua inclusão pro- gramada e assistida tem como objetivo principal a humanização das práticas assistenciais em saúde. ${ }^{(9)}$

\section{Irmãos: sua inclusão nas visitas hospitalares}

As visitas dos irmãos favorecem a integração familiar e fortalecem os laços interrompidos pela hospitalização, reduzindo os níveis de ansiedade. Por outro lado, permite o desenvolvimento da resiliência dos irmãos, dando um sentido ou significado às experiências vividas, e aproximando-os da realidade particular de cada família. Ela também facilita o surgimento de redes de apoio psico - socioafetivas tanto para as crianças quanto para seus pais. Assim, a unidade neonatal pode ser percebida como um espaço onde a família tem a possibilidade de enfrentar conjuntamente a hospitalização do recém-nascido. ${ }^{(3)}$

A inclusão da família nos diferentes centros de atendimento varia de acordo com as características estruturais, as regulamentações de cada um e as características pessoais do pessoal que labora nela. Entretanto, embora existam dificuldades relacionadas à infraestrutura e à modalidade de operação, foi demonstrado que as práticas centradas na família não estão relacionadas à disponibilidade de recursos materiais. Por outro lado, há uma grande variabilidade nas atitudes dos próprios enfermeiros. "Tem sido argumentado que cada UTIN é de certa forma semelhante a uma cultura e que em algumas existem também subculturas em diferentes rotações de enfermagem. Mudar uma cultura não é uma questão simples sem mudar atitudes e abordagens pessoais".(2)

Da mesma forma, deve haver liderança, tanto em nível hospitalar como de cada unidade, para desenvolver e apoiar políticas institucionais consistentes, a fim de evitar a variabilidade dos cuidados prestados. A criação de programas de educação e treinamento contínuos do pessoal para implementar e apoiar práticas clínicas centradas na família é uma forma de promover isto, ${ }^{(14)}$ destacando a continuidade do pessoal. A utilização destas estratégias deve ser complementar, e o contexto em que ocorre a implementação deve ser considerado. $^{(15)}$

De acordo com o acima exposto, deve-se considerar que a cultura organizacional de cada instalação tem uma influência fundamental em qualquer imple- 
mentação dos novos cuidados, pois "permite identificar barreiras e facilitadores de mudança, avaliar sua viabilidade e projetar estratégias para promovê-la e sustentá-la", avaliando por sua vez os "resultados e impactos nos processos de trabalho e relacionamentos dentro da organização". ${ }^{(16)}$ Um papel importante também é desempenhado pelo comportamento individual do pessoal de saúde e a qualidade da relação pai-provedor, influenciando diretamente a experiência dos pais durante a hospitalização. ${ }^{(17)}$

Para desenvolver isto, o papel do profissional de enfermagem é considerado essencial durante toda a internação. "A enfermeira é o elo e pilar fundamental para o vínculo entre pais e recém-nascidos durante o processo de hospitalização". ${ }^{(18)}$ Desta forma, a cultura do cuidado poderia ser um facilitador fundamental para a presença e participação dos pais, permitindo a construção de um ambiente de "cuidado neonatal" centrado na família, ${ }^{(19)}$ que está associado a impactos positivos em seus relacionamentos e bem-estar. ${ }^{(20,21)}$

Existem programas estruturados de visitação de irmãos nas áreas de terapia intensiva, a fim de aumentar os níveis de conforto do pessoal ao lidar com crianças e diminuir a resistência a eles. "Uma política de irmãos permite à enfermeira tomar decisões clínicas sólidas baseadas no conhecimento do crescimento e desenvolvimento; teoria de sistemas familiares; e literatura atual aplicada de forma hábil, solidária, consistente e individualizada, não apenas dobrando as regras quando parece justificado". (22)

Para garantir serviços de alta qualidade e integrais sob a abordagem da FCC recomenda-se fornecer recursos educacionais e emocionais aos irmãos, que podem ter a oportunidade de receber assistência direta por meio de grupos de apoio, contadores de histórias, jogos interativos e outras atividades. Uma abordagem particularmente eficaz para apoiar a compreensão dos irmãos é a história social, que narra da perspectiva do irmão o processo de hospitalização do recém-nascido, e é especialmente eficaz para crianças em idade pré-escolar e escolar. O objetivo é ser descritivo e explicativo, e visa educar os irmãos sobre a UTIN, ajudá-los a expressar seus sentimentos e aumentar sua compreensão do porquê de seus novos irmãos não poderem voltar para casa. ${ }^{(23)}$

Uma das questões que surgem com mais frequência entre os pais é que informações compartilhar com seus filhos, o que pode aumentar sua ansiedade se não for feito de maneira apropriada, portanto deve ser feito em linguagem esclarecedora de acordo com a idade e características de cada criança, verificando o que a criança já sabe."(24) Se o irmão for muito novo, os pais podem ser mais cuidadosos de como a visita afeta $o$ irmão, os pais podem influenciar-se das fantasias sobre o aparência do recém-nascido. ${ }^{(25)}$ Por outro lado, o período de tempo que a hospitalização pode durar, que varia de semanas a meses, produz um estresse progressivo na família. ${ }^{(24)}$

\section{Visitas e sua relação com a ocorrência de infecções nosocomiais}

As infecções nosocomiais são aquelas que ocorrem durante a hospitalização, que não estavam presentes ou incubadas no momento da admissão hospitalar, e estão entre as causas mais relevantes de morbidade, permanência hospitalar prolongada, aumento de custos hospitalares e mortalidade em recém-nascidos, especialmente em bebês prematuros, que estão em alto risco de exposição à infecção durante a hospitalização devido à sua condição vulnerável e à presença de micro-organismos resistentes a antibióticos, que são comuns nesses ambientes. ${ }^{(26)}$ Esse risco aumenta quanto menor for o peso e a idade gestacional ao nascer. Uma melhor sobrevivência os torna altamente suscetíveis a infecções, ${ }^{(27)}$ uma vez que a própria prematuridade está associada à maturação incompleta e/ou função do sistema imunológico. ${ }^{(28)}$

Diante do exposto, a importância de equilibrar as visitas dos irmãos com os regulamentos de controle de infecção é um tópico que tem sido revisto em alguns estudos, que sugerem a implementação de formulários que permitem a detecção oportuna de qualquer alteração no estado de saúde dos irmãos visitantes, sendo essencial o envolvimento das famílias que assumem a responsabilidade de completá-los e mantê-los atualizados. ${ }^{(29,30)}$ As visitas devem ser encorajadas, mas devem ser restritas àqueles que, independentemente de sua idade, tenham sido expostos a doenças contagiosas ou tenham sintomas de doenças infecciosas, além de verificar se os irmãos que fazem a visita estão em dia com suas imunizações. ${ }^{(31)}$ Por outro lado, é importante explicar técnicas básicas de prevenção de infecções, tais como lavagem de mãos. ${ }^{(30)}$ 


\section{Impacto da morte de um recém- nascido hospitalizado}

O processo mais triste e mais brutal de uma hospitalização é quando o recém-nascido não consegue sobreviver. Deve-se considerar o impacto de sua morte sobre os irmãos sobreviventes, pois embora a experiência possa ter um prazo relativamente curto, suas consequências podem durar uma vida inteira, tendo efeitos sobre a comunicação familiar ou aumento da ansiedade. "A assistência profissional pode ser uma poderosa adição à gama de estratégias de sobrevivência disponíveis para as famílias". ${ }^{(32)}$

A criação de memórias é uma parte importante do processo de luto, e onde os irmãos podem querer ajudar seus pais a criá-las na unidade neonatal antes ou mesmo após o momento da morte. "É importante não perder este tempo de vínculo para os irmãos, assim como para os pais e outros membros da família". (33) Até mesmo sua consideração nos serviços memoriais anuais realizados por alguns centros, poderia ser benéfico. ${ }^{(34)}$

\section{Pandemia 2020}

O cenário mundial inteiro mudou radicalmente durante o ano 2020, devido à pandemia global pelo vírus SARS Cov-2. Uma grande parte dos casos confirmados de COVID-19 foi relatada em adultos, especialmente em idosos com comorbidades. De acordo com a literatura até hoje, as crianças têm tido uma taxa relativamente mais baixa e um curso de infecção menos severo. Por sua vez, entre os pacientes pediátricos, os mais vulneráveis são os da unidade de terapia intensiva neonatal, com dados limitados sobre o efeito do vírus no nível fetal e após o nascimento. Devido a isso, os esforços têm sido concentrados no planejamento e preparação adequados a fim de proteger os pacientes, suas famílias e o pessoal de saúde. A questão da restrição de visitantes é relevante. Deve ser garantido o histórico médico dos visitantes que acredite informação da sua saúde, deve-se seguir as instruções de controle de infecção e usar o equipamento de proteção pessoal necessário. ${ }^{(35)}$ Em relação ao acima exposto, as visitas devem seguir certos protocolos que variam de acordo com a evolução da situação atual, mas que implicam em uma restrição à continuidade dos laços familiares.
Esta pandemia provavelmente adicionará mais estresse tanto para os recém-nascidos quanto para suas famílias. “Os problemas de saúde mental não são exclusivos dos pais em casa e podem afetar os irmãos e outros cuidadores". (36)

\section{Conclusão}

Os artigos revisados evidenciam a transformação que as unidades neonatais sofreram. Atualmente o foco está em melhorar a qualidade de vida do recém-nascido, mantendo a continuidade dos laços familiares durante a hospitalização, o que requer uma transformação nos papéis, sendo o profissional de enfermagem peça fundamental. As barreiras do pessoal de saúde, das famílias involucradas e das instituições devem ser consideradas, atendendo que elas podem ser superadas se tanto o pessoal quanto as famílias puderem liderar a mudança. Os aspectos mais relevantes encontrados são a detecção e redução do estresse parental e familiar, o fortalecimento dos laços entre irmãos e uma melhor comunicação familiar, o que dá espaço para a expressão dos sentimentos e preocupações que os irmãos possam ter. Por outro lado, as principais razões que impedem a realização das visitas são resumidas no perigo de transmissão de micro-organismos e infecções externas para os recém-nascidos. Também é considerado que muitas instalações de saúde têm políticas internas que impedem a entrada de crianças pequenas como visitantes em suas instalações. As evidências mostram a necessidade de implementar essas visitas como uma prioridade no cuidado com a saúde, que deve ser discutida de maneira informada e bem fundamentada, adaptando-a aos regulamentos de cada centro de saúde. As condições atuais da unidade de neonatologia, onde esta abordagem pretende ser implementada de forma otimizada, permitem seu desenvolvimento, talvez ainda sujeito à evolução da pandemia da COVID-19.

\section{Referências}

1. Sá Neto J, Rodrigues BM. Tecnologia como fundamento do cuidar em neonatologia. Texto Contexto Enfermagem. 2010;19(2):372-7.

2. Montes M, Quiroga A, Rodríguez S, Sola A. Acceso de las familias a las unidades de internación de Neonatología en Iberoamérica: una realidad a mejorar. An Pediatr. 2016;85(2):95 -101. 
Irmãos de bebês recém-nascidos internados em unidades de neonatologia: revisão bibliográfica

3. Balaguer MS, Nieto MA, Peressutti SN. Acercando las Unidades Neonatales Mediante Vínculos Saludables: Visitas Programadas de Hermanos en Neonatología. Anu investig. 2018;3(3):128-33.

4. Chile. Ministerio de Salud. Orientaciones Técnicas para la Atención Psicosocial de los Niños y Niñas Hospitalizados en Senvicios de Neonatología y Pediatría. Santiago: Ministerio de Salud, Programa de Apoyo al Desarrollo Biopsicosocial; 2011.

5. Guanuchi Y. Nivel de ansiedad en padres/madres de neonatos hospitalizados en neonatología del hospital vicente corral moscoso [tese]. Cuenca: Universidad de Cuenca, Facultad de Ciencias Médicas; 2018.

6. Larcher V. Ethical considerations in neonatal end-of-life care. Semin Fetal Neonatal Med. 2013;18(2):105 -110.

7. Janvier A, Farlow B, Baardsnes J, Pearce R, Barrington KJ. Measuring and communicating meaningful outcomes in neonatology: A family perspective. Semin Perinatol. 2016:40(8):571-7.

8. Webbe J, Brunton G, Ali S, Longford N, Modi N, Gale C. Parent, patient and clinician perceptions of outcomes during and following neonatal care: a systematic review of qualitative research. BMJ Paediatr Open. 2018;2:e000343.

9. Lozano D. Control del estrés en los padres de neonatos internados en la unidad de cuidados intensivos neonatales del servicio de neonatología-Hospital Nacional Ramiro Prialé Prialé (EsSalud Huancayo) en el período 2017- 2019 [tesis]. Huancayo: Universidad Continental, Repositorio Institucional - Continental; 2017.

10. Greisen G, Mirante N, Haumont D, Pierrat V, Pallás-Alonso C, Warren I, et al. Parents, siblings and grandparents in the Neonatal Intensive Care Unit. A survey of policies in eight European countries. Acta Paediatr. 2009;98(11):1744-50.

11. Susmani K. Certified Child Life Specialists' Perspectives On Supporting Siblings Of Infants In The Neonatal Intensive Care Unit [thesis]. Michigan: Mills College, Education; 2015.

12. Treherne S, Feeley N, Charbonneau L, Axelin A. Parents' Perspectives of Closeness and Separation With Their Preterm Infants in the NICU. J Obstet Gynecol Neonatal Nurs. 2017;46(5):737-47.

13. Franck $L S, 0^{\prime} B r i e n ~ K$. The evolution of family-centered care: From supporting parent-delivered interventions to a model of family integrated care. Birth Defects Res. 2019;111(15):104459.

14. Dall'Oglio I, Mascolo R, Tiozzo E, Portanova A, Fiori M, Gawronski 0, et al. The current practice of family-centred care in Italian neonatal intensive care units: A multicentre descriptive study. Intensive Crit Care Nurs. 2019;50:36 -43.

15. Naef R, Kläusler-Troxler M, Ernst J, Huber S, Dinten-Schmid B, Karen T, et al. Translating family systems care into neonatology practice: A mixed method study of practitioners' attitudes, practice skills and implementation experience. Int J Nurs Stud. 2020;102:103448.

16. Ramos S, Romero M, Ortiz Z, Vanessa B. Maternidad Segura y Centrada en la Familia: la cultura organizacional de maternidades de la provincia de Buenos Aires. Arch Argent Pediatr. 2015;113(6):510-8.

17. Dall'Oglio I, Fiori M, Tiozzo E, Mascolo R, Portanova A, Gawronski 0, et al. Neonata intensive care parent satisfaction: a multicenter study translating and validating the Italian EMPATHIC-N questionnaire. Ital J Pediatr. 2018;44:5.
18. Utrilla A, Sellán M, Ramos A, Mateo G. La relación enfermera - padres - neonato desde la perspectiva enfermera. Rev Cubana Enferm. 2018;34(3):637-48.

19. Aija A, Toome L, Axelin A, Raiskila S, Lehtonen L. Parents' presence and participation in medical rounds in 11 European neonatal units. Early Hum Dev. 2019;130:10-16.

20. Thomson G, Flacking R, George K, Feeley N, Haslund-Thomsen H, De Coen K, et al. Parents' experiences of emotional closeness to their infants in the neonatal unit: A metaethnography. Early Hum Dev. 2020;149:105155.

21. Flacking R, Breili C, Eriksson M. Facilities for presence and provision of support to parents and significant others in neonatal units. Acta Paediatr. 2019;108(12):2186-91.

22. Rozdilsky J. Enhancing Sibling Presence in Pediatric ICU. Crit Care Clin. 2005;17(4):451-61.

23. Morrison A, Gullón-Rivera Á. Supporting Siblings of Neonatal Intensive Care Unit Patients: A NICU Social Story ${ }^{T M}$ as an Innovative Approach. J Pediatr Nurs. 2017;33:91-3.

24. Beavis AG. What about brothers and sisters? Helping siblings cope with a new baby brother or sister in the NICU. Infant. 2007;3(6):238-42.

25. Latva R, Lehtonen L, Salmelin R, Tamminen T. Visits by the family to the neonatal intensive care unit. Acta Pædiatr. 2006;96(2):215-20.

26. Civardi E, Tzialla C, Baldanti F, Strocchio L, Manzoni P, Stronati M.Viral outbreaks in neonatal intensive care units: what we do not know. Am J Infect Control. 2013;41(10):854-6.

27. Legeay C, Bourigault C, Lepelletier D, Zahar JR. Prevention of healthcare-associated infections in neonates: room for improvement. Journal of Hospital Infection. J Hosp Infect. 2015;89(4):319-23.

28. Cortese F, Scicchitano P, Gesualdo M, Filaninno A, De Giorgi E, Schettini F, et al. Early and Late Infections in Newborns: Where Do We Stand? A Review. Pediatr Neonatol. 2016;57(4):265-73.

29. Ivany A, LeBlanc C, Grisdale M, Maxwell B, Langley JM. Reducing infection transmission in the playroom: Balancing patient safety and family-centered care.Am J Infect Control. 2016; 44(1):61-5.

30. Horikoshi Y, Okazaki K, Miyokawa S, Kinoshita K, Higuchi H, Suwa J, et al. Sibling visits and viral infection in the neonatal intensive care unit. Pediatr Int. 2018;60(2):153-6.

31. Polak JD, Ringler N, Daugherty B. Unit Based Procedures: Impact on the Incidence of Nosocomial Infections in the Newborn Intensive Care Unit. Newborn Infant Nurs Rev. 2004;4(1):38-45.

32. Fanos J, Little G, Edwards W. Candles in the Snow: Ritual and Memory for Siblings of Infants Who Died in the Intensive Care Nursery. J Pediatr. 2009;154(6):849-53.

33. Jackson C, Vasudevan C. Palliative care in the neonatal intensive care unit. Paediatr Child Health. 2020;30(4):124-8.

34. Sandler C, Robinson E, Carter B. Loss in the NICU: sibling matters. Am J Hosp Palliat Care. 2012;30(6):1-3.

35. Verma S, Lumba R, Lighter JL, Bailey SM, Wachtel EV, Kunjumon B, et al. Neonatal intensive care unit preparedness for the Novel Coronavirus Disease-2019 pandemic: A New York City hospital perspective. Curr Probl Pediatr Adolesc Health Care, 2020;50(4):100795.

36. Lemmon ME, Chapman I, Malcolm W, Kelley K, Shaw RJ, Milazzo A, et al. Beyond the First Wave: Consequences of COVID-19 on High-Risk Infants and Families. Am J Perinatol. 2020;37(12):1283-8. 